\title{
Expert Consensus on Clinical Use of an Orally Administered Dexketoprofen Plus Tramadol Fixed- Dose Combination in Moderate-To-Severe Acute Pain: A Delphi Study
}

\author{
Giustino Varrassi · Stefano Coaccioli · Josè De-Andrés · \\ Magdi Hanna · Giorgos Macheras • Antonio Montero • \\ Serge Perrot $\cdot$ Vincenzo Piras $\cdot$ Carmelo Scarpignato
}

Received: July 25, 2019 / Published online: September 18, 2019

(C) The Author(s) 2019

\section{ABSTRACT}

Introduction: In 2016, the orally administered fixed-dose combination of dexketoprofen $25 \mathrm{mg}$ and tramadol $75 \mathrm{mg}$ (DKP/TRAM FDC) was approved in Europe for short-term treatment of moderate-to-severe acute pain, an indication that encompasses a wide range of post-operative and non-surgical painful condi-

Enhanced Digital Features To view enhanced digital features for this article go to https://doi.org/10.6084/ m9.figshare.9810575.

Electronic supplementary material The online version of this article (https://doi.org/10.1007/s12325019-01096-0) contains supplementary material, which is available to authorized users.

G. Varrassi $(\bowtie)$

Paolo Procacci Foundation, Rome, Italy

e-mail: giuvarr@gmail.com

S. Coaccioli

Department of Internal Medicine, University of

Perugia, Terni, Italy

J. De-Andrés

Department of Anesthesiology Critical Care and Pain Management, General University Hospital,

Valencia, Spain

M. Hanna

Analgesics \& Pain Research Ltd, Beckenham, UK

G. Macheras

Fourth Department of Trauma and Orthopaedics,

KAT Hospital, Athens, Greece tions. This has suggested the necessity to have a clearer indication on its clinical use, with the support of expert pain clinicians, working in different medical specialities, and reinforced by the data present in the literature.

Methods: With the aim of assisting clinicians in the use of DKP/TRAM FDC in daily practice, two rounds of a modified Delphi process were conducted. In the first round, a board of nine experts developed a series of consensus statements based on available evidence, and their clinical experience, with DKP/TRAM FDC. In the second round, 75 clinicians with extensive experience in pain management expressed individually their agreement with the statements, using a dedicated online platform. Consensus was defined as at least $70 \%$ agreement.
A. Montero
Anaesthesiology and Surgery Department, Hospital
Arnau de Vilanova, Lleida, Spain
S. Perrot
Pain Center, Hôpital Cochin, Paris Descartes
University, Paris, France
V. Piras
Department of Surgical Science, University of
Cagliari, Cagliari, Italy
C. Scarpignato
Department of Clinical and Experimental Medicine, University of Parma, Parma, Italy 
Results: Twenty-eight statements were developed. Of these, 19 reached the defined level of consensus.

Conclusion: The agreed consensus statements may assist clinicians in applying the results of clinical studies and clinical experience to routine care settings, providing guidance for use of this new analgesic combination in moderate-tosevere post-operative and non-surgical acute pain.

Funding: Menarini Group.

Keywords: Acute pain; Delphi technique; Dexketoprofen; Expert opinion; Fixed-dose combination; Inflammatory pain; Moderate-tosevere pain; Pain management; Tramadol

\section{INTRODUCTION}

This decade has witnessed important advances in understanding the pathophysiologic basis of pain. The increasing implementation of standardized pain evaluation, treatment protocols and the routine use of multimodal analgesic techniques are hopeful signs that improvements in pain management are likely to continue in the years ahead [1]. Combining analgesics that act by different mechanisms of action potentially provides multimodal coverage in a broad spectrum of painful conditions and enables individual agents to act with potentially additive or synergistic effects [2]. A rational multimodal approach can also reduce the frequency of adverse effects for a better efficacy-to-safety ratio [3]. Orally administered fixed-dose analgesic combinations possibly offer additional advantages over extemporaneous combinations, including ease of administration, reduction of pill burden and potential for greater patient adherence [4].

The orally administered fixed-dose combination of dexketoprofen $25 \mathrm{mg}$ and tramadol $75 \mathrm{mg}$ (DKP/TRAM FDC) has shown an analgesic efficacy greater than that achieved by either component in monotherapy in the wellestablished human pain models of dental pain $[5,6]$, soft tissue surgery [7] and joint replacement surgery [8]. The complementary mechanisms of action that contribute to DKP/TRAM
FDC multimodal analgesia include (a) analgesic and anti-inflammatory action of dexketoprofen (and its central action); (b) opioid receptor activation by tramadol; (c) indirect activation of central descending monoaminergic pathways by tramadol, with consequent inhibition of nociceptive transmission to the brain [9].

In 2016, DKP/TRAM FDC was approved for use in Europe by a decentralized procedure. This same formulation is under registration in many countries outside of Europe. DKP/TRAM FDC is indicated for short-term symptomatic treatment of moderate-to-severe acute pain that includes a wide range of post-operative and non-surgical painful conditions.

As significant advances in the use of a medication tend to occur well after drug approval, ongoing and future long-term studies of DKP/ TRAM FDC are expected to yield important information about painful conditions that will benefit most from treatment with this new analgesic combination. However, until such data are available, a modified Delphi study appears a particularly well-suited method to develop consensus statements which reflect the collective prescribing and management patterns of experts with considerable knowledge and experience in the management of perioperative and non-surgical pain.

With the purpose of improving pain management, a panel of experts evaluated selected statements, with the Delphi method, with regard to the utilization of DKP/TRAM FDC.

\section{METHODS}

\section{Design and Identification of Experts}

The Delphi method [10] is an indirect, anonymous, iterative process aimed at achieving consensus among experts in complex scenarios where knowledge of a particular topic is available, but definitive experiments to prove the points are lacking [11]. It is a widely adopted methodology in health research in the fields of policy analysis, technology assessment, and education and training; it is also used for developing consensus statements on disease management and drug therapy [12]. 
A modified Delphi method [13] was used to generate and achieve consensus on the use of DKP/TRAM FDC in clinical practice. The definition of consensus was determined before the analysis, and it was set at at least $70 \%$ agreement [14]. Investigators were blinded to the results during analysis. The process began identifying a board of international experts with at least 15 years' experience in pain management and at least one peer-reviewed publication on analgesic combinations within the last 5 years. Nine identified experts, authors of this article, agreed to participate in the study. Between March 2017 and November 2017, two Delphi rounds were conducted. Descriptive statistical analyses were performed using SPSS ver. 23 (IBM Corp., Armonk, NY, USA).

\section{Delphi Rounds}

On the basis of available evidence about DKP/ TRAM FDC, the board of nine international experts supported the research team members in defining the main items of an open-ended questionnaire. In fact, using a dedicated online platform, the experts were asked to respond individually to open-ended questions. On the basis of the answers, 31 consensus statements were developed. Subsequently, the identified international experts met in person in June 2017. During the meeting, the members of the board commented individually on the consensus statements, indicating which of them were to be discarded. The experts were also invited to include comments and any literature data that they considered useful to propose additional statements. Plenary discussion on the revised list of statements was held. Following the meeting, a final list of 28 topically organized consensus statements was distributed by email to the board of experts for final approval. At this stage, the board members proposed only minor linguistic modifications.

In the second Delphi round, research team members defined, with the assistance of the board, a list of 118 experts in pain management (the panellists) working in centres of excellence located in clinical centres or university departments. Only clinicians with extensive experience in pain management were invited by email to participate in the Delphi process. Using a dedicated online platform, clinicians were invited to indicate their level of 'agreement' by rating each statement on a 5-point Likert-type scale (1-'strongly disagree', 2-'disagree', 3'partially agree', 4-'agree', 5-'strongly agree'). A reminder was sent to participants who had not yet responded by the given deadline. Participants' characteristics, such as gender, medical specialty, years of experience in pain management and the employment continent, were collected. The Delphi platform has been kept online for 10 weeks. No face-to-face meetings were held among panellists in order to maintain anonymity and to cast a wide geographical net. Anonymised results from round 2 circulated among the board of experts during a second face-to-face meeting. Statements for which at least $70 \%$ consensus was not achieved were reviewed and openly discussed by the experts. Plenary discussion on consensus statements was held.

The authors declare that, according to Italian law, the approval of an ethics committee is not required. The authors declare that the study is conducted in accordance with the Helsinki Declaration. The authors also declare under their own responsibility that the panel members gave their consent to participate in the study and have given consent to publish the data presented in the submitted manuscript, in an anonymous way.

\section{RESULTS}

In Delphi round 1, 28 consensus statements were defined with the assistance of the board of nine international experts in pain management. The statements were grouped into four categories: (1) pharmacological features of DKP/ TRAM, (2) clinical use of DKP/TRAM in postoperative pain management, (3) clinical use of DKP/TRAM in non-surgical pain and (4) advantages of DKP/TRAM.

In round 2 , the identified consensus statements were introduced using an online questionnaire. A panel of 75 experts responded to the e-Delphi questionnaire (response rate 
Table 1 Baseline characteristics of the panellists

\begin{tabular}{|c|c|c|}
\hline Characteristics & Number & Percentage \\
\hline \multicolumn{3}{|l|}{ Gender } \\
\hline Men & 58 & 77.3 \\
\hline Women & 17 & 22.7 \\
\hline \multicolumn{3}{|l|}{ Medical speciality } \\
\hline Anaesthesiology & 48 & 64.0 \\
\hline Orthopaedics & 9 & 12.0 \\
\hline Algology & 9 & 12.0 \\
\hline Internal medicine & 4 & 5.3 \\
\hline Dentistry & 2 & 2.7 \\
\hline Pharmacology & 1 & 1.3 \\
\hline Rheumatology & 1 & 1.3 \\
\hline Neurology & 1 & 1.3 \\
\hline \multicolumn{3}{|l|}{ Employment continent } \\
\hline Europe & 57 & 76.0 \\
\hline Central and South America & 9 & 12.0 \\
\hline Asia & 7 & 9.3 \\
\hline North America & 2 & 2.7 \\
\hline \multicolumn{3}{|c|}{ Experience in pain management, years } \\
\hline$>10$ & 67 & 89.3 \\
\hline $5-10$ & 6 & 8.0 \\
\hline$<5$ & 2 & 2.7 \\
\hline
\end{tabular}

63.6\%). As shown in Table 1, 58 responders were men and 17 women; 57 responders were based in Europe and 18 in other continents. The majority of participants were anaesthesiologists $(n=48)$. Almost $90 \%$ of panellists $(n=67)$ declared more than 10 years' experience in pain management.

In total, 19 of the 28 statements reached the defined level of consensus, as at least $70 \%$ of participants had indicated a score of 4 (agree) or 5 (strongly agree). The complete list of statements to reach consensus is provided in Table 2 . Nine statements failed to reach the minimum level of agreement and were neither accepted nor re-evaluated (Table 3). Percentages of agreement for each statement are reported in the online only supplementary materials (Tables S1 and S2).

\section{DISCUSSION}

The purpose of this Delphi study was to develop practical guidance for clinicians about the use of DKP/TRAM FDC in routine clinical practice. We used a modified Delphi method to build consensus among a panel of experts with substantial experience in pain management. The major modification to the standard Delphi technique involved originating the process with a set of pre-formulated statements developed by a group of international experts with in-depth knowledge about the use of analgesic combinations and particularly about DKP/TRAM FDC.

In this section, we present final consensus statements (Table 2) and deadlocked statements (Table 3), with supplementary comments, which reflect the reasoning behind them, based on experts' discussion, and supported by references as needed.

\section{Pharmacological Features of DKP/TRAM}

1. DKP/TRAM FDC combines two well-known drugs with proven efficacy and safety profile.

2. In moderate-to-severe acute pain, the FDC of DKP (a rapidly acting NSAID) plus TRAM (a long-lasting opioid) provides effective analgesia due to its multiple modes and sites of action.

3. In moderate-to-severe acute nociceptive somatic or visceral pain, the complementary pharmacokinetic profile of DKP with TRAM provides effective analgesia with rapid onset and long duration.

Supplementary Comment: DKP/TRAM FDC provides multimodal analgesia via different mechanisms of action in the peripheral and central nervous systems [9]. In DKP/TRAM FDC, the rapid onset of analgesic effect of dexketoprofen [15] is accompanied and complemented by the prolonged duration of action of tramadol 
Table 2 Statements that reached the defined level of consensus $(\geq 70 \%)$

Pharmacological features of the orally administered fixed-dose combination (FDC) of dexketoprofen $25 \mathrm{mg}$ and tramadol $75 \mathrm{mg}(\mathrm{DKP} / \mathrm{TRAM})$

I DKP/TRAM FDC combines two well-known drugs with proven efficacy and safety profile

II In moderate-to-severe acute pain, the FDC of DKP (a rapidly acting NSAID) plus TRAM (a long-lasting opioid) provides effective analgesia due to its multiple modes and sites of action

III In moderate-to-severe acute somatic or visceral pain, the complementary pharmacokinetic profile of DKP with TRAM provides effective analgesia with rapid onset and long duration

Clinical use of the DKP/TRAM FDC in post-operative pain management

IV DKP/TRAM FDC offers effective multimodal analgesia to treat acute post-operative pain, as recommended by the recent guidelines on the management of post-operative pain

$\mathrm{V} \quad$ In day case surgery, DKP/TRAM FDC could provide effective and rapid pain management, enabling patients to return more quickly to their normal daily activities

VI In major abdominal surgery such as hysterectomy for benign conditions/gynaecological abdominal surgery, DKP/ TRAM FDC has represented an effective treatment of moderate-to-severe acute post-operative pain

VII In major orthopaedic surgery such as hip or knee replacement, the efficacy of DKP/TRAM FDC in controlling post-operative pain both at rest and on movement could allow early patient mobilization, lowering thromboembolic risk, shortening hospitalization stay and enabling a better rehabilitation

VIII The use of the orally administered DKP/TRAM FDC is likely to be envisaged for minor orthopaedic surgeries such as rotator cuff repair, arthroscopic shoulder and knee surgeries, bunionectomy (hallux valgus surgery), meniscectomy, release of the median nerve and fracture management

IX DKP/TRAM FDC is likely to be an effective analgesic in minor surgeries such as appendectomies, endoscopic bladder surgery, endoscopic prostatectomy (TURP), plastic surgery of soft parts, rhinoplastic, endoscopic video thoracic surgery, hernia repair, cholecystectomy, ocular surgery, skin surgery, abscess drainage

$\mathrm{X} \quad \mathrm{DKP} / \mathrm{TRAM}$ FDC has provided effective and rapid post-operative pain relief in surgical removal of impacted wisdom teeth and dental surgeries

Clinical use of the DKP/TRAM FDC in non-surgical pain

XI DKP/TRAM FDC is likely to provide a good mean for effective pain management in moderate-to-severe acute dental pain due to its shorter time to onset of analgesia and its anti-inflammatory activity

XII Owing to its pharmacological profile, DKP/TRAM FDC could be an important tool in controlling moderate-tosevere pain in acute exacerbations of osteoarticular pain where nociceptive and neuropathic mechanisms are involved in both the local and central levels

XIII Owing to its pharmacological profile, DKP/TRAM FDC will probably have an important role in pain control in acute exacerbations of low back pain since both components (DKP \& TRAM) have proven efficacy, and where nociceptive and neuropathic mechanisms are involved at both local and central levels

XIV DKP/TRAM FDC, targeting different sites of action, is suitable for mixed types of pain, arising from different body structures (joints, muscles, ligaments, etc.) such as acute articular pain or non-specific low back pain 
Table 2 continued

XV DKP/TRAM FDC, owing to its multiple modes and sites of action, could be an important addition in the control of post-traumatic pain with somatic and neuropathic component (e.g. soft tissue trauma, dislocated long bone fractures, extremities fractures)

Advantages of the DKP/TRAM FDC

XVI DKP/TRAM FDC offers additional advantages over non-fixed combinations, including ease of administration, reduction of pill burden and will improve adherence by patients

XVII The oral route of administration of DKP/TRAM FDC improves patient compliance and adherence to treatment and will be of advantage in the outpatient setting

XVIII DKP/TRAM FDC could be of great value when other single or combined drugs were less efficacious or could not be tolerated because of adverse side effects

XIX In moderate-to-severe acute pain, the DKP/TRAM FDC generates effective and rapid analgesia reducing burden of pain. This in turn may potentially have a positive impact on psychosocial aspects of acute pain such as psychological vulnerability, stress, higher healthcare utilization and reduced labour force participation

[7]. Of note, a recent Cochrane review [16] on single-dose DKP/TRAM FDC for acute post-operative pain found that "a single oral dose of dexketoprofen $25 \mathrm{mg}$ plus tramadol $75 \mathrm{mg}$ provided good levels of pain relief with long duration of action to more people than the same dose of dexketoprofen or tramadol alone".

\section{Clinical Use of DKP/TRAM in Post- Operative Pain Management}

1. DKP/TRAM FDC offers effective multimodal analgesia to treat acute post-operative pain, as recommended by the recent guidelines on the management of post-operative pain.

2. In day case surgery, DKP/TRAM FDC could provide effective and rapid pain management, enabling patients to return more quickly to their normal daily activities.

3. In major abdominal surgery such as hysterectomy for benign conditions/gynaecological abdominal surgery, DKP/TRAM FDC has represented an effective treatment of moderate-to-severe acute post-operative pain.

4. In major orthopaedic surgery such as hip or knee replacement, the efficacy of DKP/
TRAM FDC in controlling post-operative pain both at rest and on movement could allow early patient mobilization, lowering thromboembolic risk, shortening hospitalization stay and enabling a better rehabilitation.

5. The use of the orally administered DKP/ TRAM FDC is likely to be envisaged for minor orthopaedic surgeries such as rotator cuff repair, arthroscopic shoulder and knee surgeries, bunionectomy (hallux valgus surgery), meniscectomy, debridement of the median nerve and fracture management.

6. DKP/TRAM FDC is likely to be an effective analgesic in minor surgeries such as appendectomies, endoscopic bladder surgery, endoscopic prostatectomy (TURP), plastic surgery of soft parts, rhino-plastic, endoscopic video thoracic surgery, hernia repair, laparoscopic cholecystectomy, ocular surgery, skin surgery, abscess drainage.

7. DKP/TRAM FDC has provided effective and rapid post-operative pain relief in surgical removal of impacted wisdom teeth and dental surgeries.

Supplementary Comment: In well-designed, randomized clinical trials $[5,8]$, DKP/TRAM 
Table 3 Statements which failed to achieve at least $70 \%$ consensus

Pharmacological features of the orally administered fixed-dose combination (FDC) of dexketoprofen $25 \mathrm{mg}$ and tramadol $75 \mathrm{mg}$ (DKP/TRAM)

a DKP/TRAM FDC provides a good means for effective analgesia at reduced doses of individual agents with a potential reduction of opioid dose-related adverse events such as constipation, respiratory depression, nausea and vomiting

b Safety profile of DKP/TRAM FDC is fully in line with that previously known for the single agents in monotherapy

Clinical use of the DKP/TRAM FDC in post-operative pain management

c DKP/TRAM FDC has a potential advantage in moderate-to-severe acute post-operative pain owing to its rapid onset of action. This in turn may reduce risks of medical complications and of developing chronic pain, allowing a fast hospital discharge and a more effective outpatient pain management

d DKP/TRAM FDC will provide effective analgesia in major surgeries, when the oral route (or the switch to it) is feasible

Clinical use of the DKP/TRAM FDC in non-surgical pain

e The orally administered DKP/TRAM FDC provides effective analgesia in moderate-to-severe acute symptomatic painful conditions and its use is likely to be shown as beneficial in a wide range of non-surgical clinical conditions

f In acute cervical pain, DKP/TRAM FDC through very effective analgesia may allow one to reduce the muscular tension and the consequent spasmodic pain

g DKP/TRAM FDC has the potential to be useful for renal colic pain and dysmenorrhoea, a distressing condition with important visceral component and particularly for secondary dysmenorrhoea caused by endometriosis, since the two components (DKP \& TRAM) have proven efficacy in those pain syndromes

Advantages of the DKP/TRAM FDC

$\mathrm{h}$ In specific pain models, less rescue medications were needed when the DKP/TRAM FDC was used

i Compared to the single agents in monotherapy, DKP/TRAM FDC offers potential benefits such as longer time to remedication and better patient global evaluation

FDC was effective in moderate-to-severe postoperative acute pain management. It was also superior to the combination paracetamol/tramadol (650/75 mg) [6].

In major surgeries such as hip replacement, DKP/TRAM FDC showed superior and sustained efficacy for post-operative pain control compared with individual components at the same (i.e. dexketoprofen $25 \mathrm{mg}$ ) or higher (i.e. tramadol $100 \mathrm{mg}$ ) doses. This, in turn, facilitates early patient mobilization, lowering thromboembolic risk, shortening hospitalization and timely starting of rehabilitation [17].

DKP/TRAM FDC can also be envisaged for use in a wide range of minor or day case surgeries. As an increasing number and complex elective operations are being performed on an ambulatory (or short-stay) basis, effective multimodal analgesic regimens that can be managed successfully at home by patients and their caregivers are required [1].

\section{Clinical Use of DKP/TRAM in Non-Surgical Pain}

1. DKP/TRAM FDC is likely to provide a good means for effective pain management in moderate-to-severe acute dental pain due to its shorter time to onset of analgesia and its anti-inflammatory activity.

2. Owing to its pharmacological profile, DKP/ TRAM FDC could be an important tool in 
controlling moderate-to-severe pain in acute exacerbations of osteoarticular pain where nociceptive and neuropathic mechanisms are involved in both the local and central levels.

3. Owing to its pharmacological profile, DKP/ TRAM FDC will probably have an important role in pain control in acute exacerbations of low back pain since both components (DKP \& TRAM) have proven efficacy, and where nociceptive and neuropathic mechanisms are involved at both local and central levels.

4. DKP/TRAM FDC, targeting different sites of action, is suitable for mixed types of pain arising from different body structures (joints, muscles, ligaments, etc.) such as acute articular pain or non-specific low back pain.

5. DKP/TRAM FDC, owing to its multiple modes and sites of action, could be an important addition in the control of posttraumatic pain with somatic and neuropathic component (e.g. soft tissue trauma, dislocated long bone fractures, extremities fractures).

Supplementary Comment: Recent systematic reviews [18] and clinical recommendations [19] focus primarily on post-operative acute pain although non-surgical acute pain (e.g. posttrauma pain or acute flares of osteoarticular pain) should also be addressed. DKP/TRAM FDC provided effective post-operative pain control after third mandibular molar tooth extraction [5], which is a model of acute pain frequently used to investigate the pharmacodynamics properties of analgesic molecules (i.e. onset/ offset, dose-response and potency) [20]. Efficacy in dental model is highly predictive of efficacy in later stage models [21]. Furthermore, third molar surgery is a common procedure with sufficient patient numbers to make these studies relatively easy to perform [22]. In patients with moderate-to-severe acute dental pain, the analgesic and anti-inflammatory activity of orally administered dexketoprofen trometamol was found to be similar to that of ibuprofen, with a shorter time to onset of analgesia [23]. On these bases, DKP/TRAM FDC is envisaged for use to relieve moderate-to-severe non-surgical acute dental pain. DKP/TRAM FDC also represents an interesting option for acute exacerbations of osteoarticular pain owing to its pharmacological profile and multimodal approach [9]. Of note, osteoarticular pain includes nociceptive and neuropathic mechanisms that are involved in both the local and central levels and may present with different clinical features: constant and intermittent pain, with or without a neuropathic component, and with or without central sensitization [24, 25]. By targeting different sites of action, DKP/TRAM FDC is particularly suitable for osteoarticular pain that arises from different body structures such as joints and muscles [9].

\section{Advantages of DKP/TRAM}

1. DKP/TRAM FDC offers additional advantages over non-fixed combinations, including ease of administration, reduction of pill burden and will improve adherence by patients.

2. The oral route of administration of DKP/ TRAM FDC improves patient compliance and adherence to treatment and will be of advantage in the outpatient setting.

3. DKP/TRAM FDC could be of great value when other single or combined drugs were less efficacious or could not be tolerated because of adverse side effects.

4. In moderate-to-severe acute pain, the DKP/ TRAM FDC generates effective and rapid analgesia reducing burden of pain. This in turn may potentially have a positive impact on psychosocial aspects of acute pain such as psychological vulnerability, stress, higher healthcare utilization and reduced labour force participation.

Supplementary Comment: We strongly advocate for the use of fixed-dose analgesic combinations such as DKP/TRAM FDC in the management of moderate-to-severe acute pain. In particular, orally administered fixed-dose analgesic combinations may improve patient adherence to treatments [4].

In recent years, the work-, social-, and family-related consequences of poorly managed 
pain have been extensively investigated $[26,27]$. In related fashion, the implications of inadequate acute pain management may affect negatively on individual stress, healthcare resource utilization and labour force participation [27]. All this implies that acute moderateto-severe pain must be treated as soon and as efficaciously possible.

\section{Statements for Which at Least 70\% Consensus Was Not Achieved}

(a) DKP/TRAM FDC provides a good means for effective analgesia at reduced doses of individual agents with a potential reduction of opioid dose-related adverse events such as constipation, respiratory depression, nausea and vomiting.

(b) Safety profile of DKP/TRAM FDC is fully in line with that previously known for the single agents in monotherapy.

(c) DKP/TRAM FDC has a potential advantage in moderate-to-severe acute post-operative pain owing to its rapid onset of action. This in turn may reduce risks of medical complications and of developing chronic pain, allowing a fast hospital discharge and a more effective outpatient pain management.

(d) DKP/TRAM FDC will provide effective analgesia in major surgeries, when the oral route (or the switch to it) is feasible.

(e) The orally administered DKP/TRAM FDC provides effective analgesia in moderateto-severe acute symptomatic painful conditions and its use is likely to be shown as beneficial in a wide range of non-surgical clinical conditions.

(f) In acute cervical pain, DKP/TRAM FDC through very effective analgesia may allow one to reduce the muscular tension and the consequent spasmodic pain.

(g) DKP/TRAM FDC has the potential to be useful for renal colic pain and dysmenorrhoea, a distressing condition with important visceral component and particularly for secondary dysmenorrhoea caused by endometriosis, since the two components (DKP and TRAM) have proven efficacy in those pain syndromes. (h) In specific pain models, less rescue medications were needed when the DKP/TRAM FDC was used.

(i) Compared to the single agents in monotherapy, DKP/TRAM FDC offers potential benefits such as longer time to re-medication and better patient global evaluation.

Supplementary Comment: The main premise of opioid-containing analgesic combinations is to decrease the consumption of opioids, and hence reduce the frequency of opioid-related adverse events [19]. More research is needed to evaluate if the use of the DKP/TRAM FDC is associated with a reduction of opioid dose-related adverse events.

The percentage of agreement for statement (c) fell slightly below the predefined level of agreement. Of note, recent studies [28-32] indicate that poorly managed post-operative pain can result in chronic pain. Furthermore, according to results of a cross-sectional survey on a sample of 1030 patients [33], post-operative chronic pain affects $38 \%$ of patients undergoing total hip arthroplasty. More research on the role of DKP/TRAM FDC in preventing the development of chronic pain is needed.

Likewise, statement (d) did not reach consensus. In this regard, the 2016 guideline from the American Pain Society and the American Society of Regional Anesthesia and Pain Medicine for management of post-operative pain [19] strongly recommends oral over intravenous administration of opioids for post-operative analgesia in patients able to use the oral route.

Despite the availability of scientific literature, statement (h) did not reach the predefined level of agreement. In pivotal studies of DKP/ TRAM FDC [5, 7], patients who received the analgesic combination experienced longer time to first use of rescue medication in comparison with single agents DKP $25 \mathrm{mg}$ and TRAM $100 \mathrm{mg}$. This suggests that findings from clinical trials diffuse slowly into clinical practice, highlighting the need to bridge the gap between research and practice. 


\section{Strengths and Limitations of the Study}

Our study has several strengths. Rather than begin the Delphi procedure with an open question and invite suggestions from panellists, the list of consensus statements they voted on was derived from the existing clinical literature and formulated by a board of international experts with strong expertise in pain management, and especially in the use of analgesic combinations, including DKP/TRAM FDC. During the first face-to-face meeting, experts could explain and defend their own ideas, potentially improving the quality of the initial draft of consensus statements. The sample size of panellists was relatively large compared with other Delphi studies, and electronic data collection allowed high clinician participation with a wider geographical net.

The study also had some limitations. The potential sample of panellists was large and comprised experienced people from different geographical areas; some panellists were based in countries where DKP/TRAM FDC was not released yet or not in widespread use.

For expected adverse events related to the drug, please refer to the summary of product characteristics of DKP/TRAM FDC [34].

\section{CONCLUSIONS}

DKP/TRAM FDC is a rapidly acting, long-lasting, multimodal analgesic combination that is effective and generally well tolerated in patients with moderate-to-severe acute pain [9].

Additional long-term clinical studies are expected to identify acute painful conditions that will benefit most from treatment with DKP/ TRAM FDC. Meanwhile, the consensus statements may help clinicians applying the results of clinical studies and clinical recommendations to routine-care settings, thus providing guidance for use of this new analgesic combination in moderate-to-severe post-operative and non-surgical acute pain.

\section{ACKNOWLEDGEMENTS}

The authors would like to thank the following panel members for their answers to the survey: Aguilar JL, Aréchiga GE, Battelli D, Bedran H, Benhamou D, Bhaskar AK, Bonnet F, Carrascosa A, Casale R, Colini Baldeschi G, Collighan N, Cooper A, Coupe M, Cryer B, De Bastiani R, De Negri P, Deleens R, Dias Asis F, Drewes Asbjørn $\mathrm{M}$, Erdine S, Espinoza V, and the panel members: Aguilar JL, Aréchiga GE, Battelli D, Bedran $\mathrm{H}$, Benhamou D, Bhaskar AK, Bonnet F, Carrascosa A, Casale R, Colini Baldeschi G, Collighan $\mathrm{N}$, Cooper A, Coupe $\mathrm{M}$, Cryer B, De Bastiani R, De Negri P, Deleens R, Dias Asis F, Drewes Asbjørn M, Erdine S, Espinoza VI, Esteve N, Fenech C, Fitzcharles MA, Gambopoulou Z, García AM, Hernandez JR, Hodgkinson V, Hungin P, Kamble P, Karras A, Kim Yong Chul, Knaggs R, Kouroukli I, Krishnan Manian M, Kulichova M, Lanas A, Laroche F, Licheri L, Likar R, Lo Bianco G, Maculé España F, Malik D, Mascolo A, Mejia F, Micheli F, Montes A, Moon Jee, Mugabure B, Narvaetz M A, Notcutt W, Oteo A, Pace C, Paladini A, Papadopoulos G, Peppin J, Pergolizzi J, Pickering G, Plancarte R, Polati E, Ramni S, Rawal N, Rudiger J, Salem S, Sharma A, Sittl R, Moliner S, Tharakan L, Timberlake C, Tokgozoglu M, Toledo V, Tölle T, Vadalouca A, Van Zundert J, Vélez Alvaro V.

Funding. The study has been realized thanks to an unconditioned grant of the Menarini Group. Menarini Group also funded the editorial assistance as well as Rapid Service and Open Access fees.

Editorial Assistance. Dr. Francesca Gori of Content Ed Net provided editorial assistance in the preparation of this article and was funded by the Menarini Group.

Authorship. All named authors meet the International Committee of Medical Journal Editors (ICMJE) criteria for authorship for this article, take responsibility for the integrity of the work as a whole, and have given their approval for this version to be published. 
Disclosures. Giustino Varrassi received a grant from the Menarini Group, as the main investigator of this study. He is also consultant for the following companies: Abbott, BerlinChemie, Dompé, Menarini Asia-Pacific, Molteni, and Shionogi. Giustino Varrassi is the Pain Section Editor for Advances in Therapy. Stefano Coaccioli received a grant from the Menarini Group for participation in this study, as an international expert. Josè De-Andrés received a grant from the Menarini Group for participation in this study, as an international expert. Magdi Hanna received a grant from the Menarini Group for participation in this study, as an international expert. Giorgos Macheras received a grant from the Menarini Group for participation in this study, as an international expert. Antonio Montero received a grant from the Menarini Group for participation in this study, as an international expert. Serge Perrot received a grant from the Menarini Group for participation in this study, as an international expert. Vincenzo Piras received a grant from the Menarini Group for participation in this study, as an international expert. Carmelo Scarpignato received a grant from the Menarini Group for participation in this study, as an international expert. Carmelo Scarpignato also declares that he has served as a speaker, consultant and/or advisory board member for Alfasigma, Pfizer, Takeda, Reckitt-Benkiser and Shionogi and has in the past received funding from Giuliani Pharmaceuticals and Pfizer.

Compliance with Ethics Guidelines. The authors declare that, according to Italian law, the approval of an ethics committee is not required. The authors declare that the study is conducted in accordance with the Helsinki Declaration. The authors also declare, under their own responsibility, that the panel members gave their consent to participate in the study and have given consent to publish the data presented in the submitted manuscript, in an anonymous way.

Data Availability. The database analyzed during the current study is available from the corresponding author on reasonable request.
Open Access. This article is distributed under the terms of the Creative Commons Attribution-NonCommercial 4.0 International License (http://creativecommons.org/licenses/ by-nc/4.0/), which permits any noncommercial use, distribution, and reproduction in any medium, provided you give appropriate credit to the original author(s) and the source, provide a link to the Creative Commons license, and indicate if changes were made.

\section{REFERENCES}

1. Elvir-Lazo OL, White PF. The role of multimodal analgesia in pain management after ambulatory surgery. Curr Opin Anaesthesiol. 2010;23(6):697-703.

2. Raffa RB, Tallarida RJ, Taylor R Jr, Pergolizzi JV Jr. Fixed-dose combinations for emerging treatment of pain. Expert Opin Pharmacother. 2012;13(9):1261-70.

3. Raffa RB, Pergolizzi JV Jr, Tallarida RJ. The determination and application of fixed-dose analgesic combinations for treating multimodal pain. J Pain. 2010;11(8):701-9.

4. O’Brien J, Pergolizzi J, van de Laar M, et al. Fixeddose combinations at the front line of multimodal pain management: perspective of the nurse-prescriber. Nurs Res Rev. 2013;3:9-22.

5. Moore RA, Gay-Escoda C, Figueiredo R, et al. Dexketoprofen/tramadol: randomised double-blind trial and confirmation of empirical theory of combination analgesics in acute pain. J Headache Pain. $2015 ; 16: 541$.

6. Gay-Escoda C, Hanna M, Montero A, et al. Tramadol/dexketoprofen (TRAM/DKP) compared with tramadol/paracetamol in moderate to severe acute pain: results of a randomised, double-blind, placebo and active-controlled, parallel group trial in the impacted third molar extraction pain model (DAVID study). BMJ Open. 2019;9(2):e023715.

7. Moore RA, McQuay HJ, Tomaszewski J, et al. Dexketoprofen/tramadol $25 \mathrm{mg} / 75 \mathrm{mg}$ : randomised double-blind trial in moderate-to-severe acute pain after abdominal hysterectomy. BMC Anesthesiol. 2016;16:9.

8. McQuay HJ, Moore RA, Berta A, et al. Randomized clinical trial of dexketoprofen/tramadol $25 \mathrm{mg} /$ $75 \mathrm{mg}$ in moderate-to-severe pain after total hip arthroplasty. Br J Anaesth. 2016;116:269-76. 
9. Varrassi G, Hanna M, Macheras G, et al. Multimodal analgesia in moderate-to-severe pain: a role for a new fixed combination of dexketoprofen and tramadol. Curr Med Res Opin. 2017;33(6):1165-73.

10. Jones J, Hunter D. Consensus methods for medical and health services research. BMJ. 1995;311:376-80.

11. Durand MA, Chantler R. Consensus groups in principles of social research. Maidenhead: Open University Press/McGraw-Hill; 2014. p. 64-6.

12. Keeney S, Hasson F, Mckenna H. The Delphi technique in nursing and health research. Chichester: Wiley-Blackwell; 2011. p. 208.

13. Keeney S, Hasson F, Mckenna H. The Delphi technique in nursing and health research. Chichester: Wiley; 2010. p. 55.

14. Diamond IR, Grant RC, Feldman BM, et al. Defining consensus: a systematic review recommends methodologic criteria for reporting of Delphi studies. J Clin Epidemiol. 2014;67(4):401-9.

15. McGurk M, Robinson P, Rajayogeswaran V, et al. Clinical comparison of dexketoprofen trometamol, ketoprofen, and placebo in postoperative dental pain. J Clin Pharmacol. 1998;38(12 Suppl):46S-54S.

16. Derry S, Cooper TE, Phillips T. Single fixed-dose oral dexketoprofen plus tramadol for acute postoperative pain in adults. Cochrane Database Syst Rev. 2016;9:CD012232.

17. Young AC, Buvanendran A. Pain management for total hip arthroplasty. J Surg Orthopaed Adv. 2014;23:13-21.

18. Au AH, Choi SW, Cheung CW, Leung YY. The efficacy and clinical safety of various analgesic combinations for post-operative pain after third molar surgery: a systematic review and meta-analysis. PLoS One. 2015;10(6):e0127611.

19. Chou R, Gordon DB, de Leon-Casasola OA, et al. Management of postoperative pain: a clinical practice guideline from the American Pain Society, the American Society of Regional Anesthesia and Pain Medicine, and the American Society of Anesthesiologists' Committee on Regional Anesthesia, Executive Committee, and Administrative Council. J Pain. 2016;17:131-57.

20. Cooper SA, Desjardins PJ. The value of the dental impaction pain model in drug development. Methods Mol Biol. 2010;617:175-90.

21. Singla NK, Desjardins PJ, Chang PD. A comparison of the clinical and experimental characteristics of four acute surgical pain models: dental extraction, bunionectomy, joint replacement, and soft tissue surgery. Pain. 2014;155(3):441-56.

22. Barden J, Edwards JE, McQuay HJ, Moore AR. Pain and analgesic response after third molar extraction and other postsurgical pain. Pain. 2004;107(1-2):86-90.

23. Mauleon D, Artigas R, Garcia ML, et al. Preclinical and clinical development of dexketoprofen. Drugs. 1996;52(Suppl 5):24-45 (discussion 45-46).

24. Perrot S. Osteoarthritis pain. Best Pract Res Clin Rheumatol. 2015;29:90-7.

25. Fusco M, Skaper S, Coaccioli S, Paladini A, Varrassi G. Degenerative joint diseases and neuroinflammation. Pain Pract. 2017;17:522-32.

26. Dueñas M, Ojeda B, Salazar A, Mico JA, Failde I. A review of chronic pain impact on patients, their social environment and the health care system. J Pain Res. 2016;28(9):457-67.

27. Langley P, Mueller-Schwefe G, Nicolau A, Liedgens H, Pergolizzi J, Varrassi G. The societal impact of pain in European Union: health-related quality of life and healthcare resource utilization. J Med Econ. 2010;13:571-81.

28. Jin J, Peng L, Chen Q, et al. Prevalence and risk factors for chronic pain following cesarean section: a prospective study. BMC Anesthesiol. 2016;16:99.

29. Weibel S, Neubert K, Jelting Y, et al. Incidence and severity of chronic pain after caesarean section: a systematic review with meta-analysis. Eur J Anaesthesiol. 2016;33:853-65.

30. Gerbershagen HJ, Dagtekin O, Rothe T, et al. Risk factors for acute and chronic postoperative pain in patients with benign and malignant renal disease after nephrectomy. Eur J Pain. 2009;13:853-60.

31. Beswick AD, Wylde V, Gooberman-Hill R, et al. What proportion of patients report long-term pain after total hip or knee replacement for osteoarthritis? A systematic review of prospective studies in unselected patients. BMJ Open. 2012;2:e000435.

32. Simanski CJ, Althaus A, Hoederath S, et al. Incidence of chronic postsurgical pain (CPSP) after general surgery. Pain Med. 2014;15:1222-9.

33. Liu SS, Buvanendran A, Rathmell JP, et al. A crosssectional survey on prevalence and risk factors for persistent postsurgical pain 1 year after total hip and knee replacement. Reg Anesth Pain Med. 2012;37:415-22.

34. Skudexa $75 \mathrm{mg} / 25 \mathrm{mg}$ summary of product characteristics. https://www.medicines.org.uk. Accessed 30 June 2019. 